\title{
Digital live-tracking 3-dimensional minisensors for recording head orientation during image acquisition
}

\author{
Leonardo Koerich de Paula ${ }^{a}$, James L. Ackerman ${ }^{b}$, Felipe de Assis Ribeiro Carvalhoc, \\ Lindsey Eidson ${ }^{d}$, and Lucia Helena Soares Cevidanes ${ }^{e}$ \\ ${ }^{a}$ Research assistant, Department of Orthodontics, School of Dentistry, University of North \\ Carolina, Chapel Hill

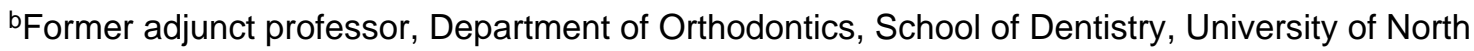 \\ Carolina, Chapel Hill \\ cPostgraduate student, Department of Orthodontics, School of Dentistry, State University of Rio \\ de Janeiro, Rio de Janeiro, Brazil \\ ${ }^{\mathrm{d}}$ Resident, Department of Orthodontics, School of Dentistry, University of North Carolina, Chapel \\ Hill \\ eAssistant professor, Department of Orthodontics, School of Dentistry, University of North \\ Carolina, Chapel Hill
}

\begin{abstract}
Introduction-Our objective was to test the value of minisensors for recording unrestrained head position with 6 degrees of freedom during 3-dimensional stereophotogrammetry.

Methods-Four 3-dimensional pictures (3dMD, Atlanta, Ga) were taken of 20 volunteers as follows: (1) in unrestrained head position, (2) a repeat of picture 1, (3) in unrestrained head position wearing a headset with 3-dimensional live tracking sensors (3-D Guidance trackSTAR; Ascension Technology, Burlington, Vt), and (4) a repeat of picture 3. The sensors were used to track the $\mathrm{x}, \mathrm{y}$, and $\mathrm{z}$ coordinates (pitch, roll, and yaw) of the head in space. The patients were seated in front of a mirror and asked to stand and take a walk between each acquisition. Eight landmarks were identified in each 3-dimensional picture (nasion, tip of nose, subnasale, right and left lip commissures, midpoints of upper and lower lip vermilions, soft-tissue B-point). The distances between correspondent landmarks were measured between pictures 1 and 2 and 3 and 4 with software. The Student $t$ test was used to test differences between unrestrained head position with and without sensors.
\end{abstract}

Results-Interlandmark distances for pictures 1 and 2 (head position without the sensors) and pictures 3 and 4 (head position with sensors) were consistent for all landmarks, indicating that roll, pitch, and yaw of the head are controlled independently of the sensors. However, interlandmark distances were on average $17.34 \pm 0.32 \mathrm{~mm}$ between pictures 1 and 2 . Between pictures 3 and 4 , the distances averaged $6.17 \pm 0.15 \mathrm{~mm}$. All interlandmark distances were significantly different between the 2 methods $(P<0.001)$.

Conclusions-The use of 3-dimensional live-tracking sensors aids the reproducibility of patient head positioning during repeated or follow-up acquisitions of 3-dimensional

Copyright $\odot 2012$ by the American Association of Orthodontists.

Reprint requests to: Leonardo Koerich de Paula, Rua Tenente Olimpio Costa n114, Apt 205 B, Florianopolis, SC, Brazil 88090-080; lkoerich@gmail.com..

The authors report no commercial, proprietary, or financial interest in the products or companies described in this article. 
stereophotogrammetry. Even with sensors, differences in spatial head position between acquisitions still require additional registration procedures.

The relatively recent transition in orthodontics from 2-dimensional (2D) to 3-dimensional (3D) imaging and from analog to digital technology has created renewed impetus for finding a versatile method for establishing accurate and reliable head positioning during the acquisition of serial records. During the analog era, orthodontists used cephalostats to orient radiographic films for diagnosis and tracking longitudinal changes from either growth or treatment. In the digital era, head orientation for the virtual patient presents a new challenge. When viewing a digital image on a computer screen, there is no external reference to establish the natural orientation of the head and teeth. Cone-beam computerized tomography (CBCT) and 3D photographic imaging offer new possibilities for more comprehensive diagnosis and treatment planning in clinical orthodontics because they give far more information than the previous $2 \mathrm{D}$ records. However, additional tools are required to achieve accuracy and reliability in the capture of these images, and proper orientation is important for future superimposition of the images to assess changes. Matching records taken at 2 times requires a more complex computer registration than was heretofore necessary. This research project addressed the issue of recording head position when it is unrestrained as it is during image capture for 3D photography or CBCT.

Head orientation has been a subject of great interest for clinical and research orthodontists for more than a century. In 1882, the General Congress of the German Anthropology Society agreed on a standard skull orientation proposed by Von Ihering. ${ }^{1,2}$ His suggestion was that a line, named the Frankfort horizontal, extending from the upper ridge of the external auditory meatus to the most inferior portion of the orbit should be parallel to the floor. This strictly anatomic method of skull orientation could only be used for dry skulls, plaster facial moulages, and dental casts. ${ }^{3}$ When Broadbent ${ }^{4}$ and Hofrath ${ }^{5}$ proposed radiographic cephalometry in 1931, for the first time it was possible to study living heads; because of their proposed stereotactic head-holding device, the cephalometer, it was possible to study facial growth longitudinally. The positioning of the head in the cephalostat initially chosen for this purpose was the orientation proposed by Von Ihering-the Frankfort horizontal. However, in 1956, Downs, ${ }^{6}$ using photographs, demonstrated variations in the Frankfort horizontal plane when subjects were in natural head posture. This prompted Moorrees and Kean, ${ }^{7}$ in 1958, to introduce in the orthodontic literature a physiologic position, natural head position. Natural head position is determined largely by the visual axis and can be obtained by having the subject stand and look at the horizon. Alternatively, a mirror can be placed in front of the subject and ask him or her to look at the eyes in the mirror. Natural head position was originally proposed by $\mathrm{Broca}^{8}$ to replace the anatomic method with the Frankfort horizontal plane. ${ }^{7}$ Vig et al $^{9}$ observed changes in natural head position as a result of respiratory obstruction, and discussed that posture and respiration had implications in the control of growth and the establishment of dentofacial morphology.

Several authors tested the reliability of natural head position in 2 dimensions using lateral cephalometric radiographs. ${ }^{7,10-15}$ Others used photographs in addition to cephalograms to help in testing the reproducibility of natural head position. ${ }^{16,17}$ In all of these studies, the evaluations and comparisons of natural head position were with 2D images. The reproducibility of natural head position was evaluated for the capture of 3D images by Xia et al, ${ }^{16}$ who achieved natural head position for stereolithographic skull models of patients with dentofacial deformities with 2 methods. The first consisted of a laser scan capturing the facial soft-tissues' surface and matching it with a rendered composite skull model. The second technique used a gyroscope attached to a face-bow that provided the pitch, yaw, and roll angulations of the patient's head, which was used to reorient the skull on the computer. The reproducibility of head position in 3 dimensions was also studied by Soncul and 
Bamber. ${ }^{17}$ Using a facial laser scan, a headrest, and a spirit level, they showed high accuracy in reproducing head orientation; however, the Frankfort plane was used rather than natural head position.

The objective of this study was to test the effectiveness of minisensors in achieving repeatable head positioning. Because currently available imaging equipment is not designed for a standing patient, it was necessary to have our test subjects sit, rather than stand, although a mirror was used in front of them to simulate natural head position. The ultimate goal of this project was to redesign the imaging equipment, if necessary, and use the $3 \mathrm{D}$ sensors to record natural head position.

\section{MATERIAL AND METHODS}

Twenty volunteers (13 men, 7 women; mean age, $32.80 \pm 8.7$ years; range, 20.3-55.6 years) were selected for this study. The exclusion criteria were (1) dentofacial deformities, (2) facial hair, (3) orthodontic appliances, (4) clinical diagnosis of asymmetry, (5) pacemaker, and (6) lip incompetence. The protocol was approved by the Biomedical Institutional Review Board of the University of North Carolina, and informed consent was obtained from all subjects.

Three-dimensional surface images of each patient were acquired with the $3 \mathrm{dMD}$ face system (3dMD, Atlanta, Ga) on the same day (Fig 1) in 4 situations: (1) the patient in unrestrained head position, (2) repeated picture with the patient in unrestrained head position, (3) the patient in unrestrained head position wearing a headset with tracking sensors, and (4) repeated picture of the patient in unrestrained head position wearing a headset with tracking sensors (Fig 2).

The seat for the 3D photograph acquisition was a chair with (1) the ability to adjust the seat's vertical height to accommodate subjects of varying heights and (2) a back support to help the subjects simulate their standing posture. Because the subnasal and submental regions are prone to data loss and artefacts, proper head posture ensured that these regions were visible to the imaging sensors of the camera. If the subject's head tilted forward even a few degrees, these facial regions were often obliterated, and it was necessary to remind the subject to sit up straight. In addition to obvious signs of facial tension (eg, furrowed brow) or emotional expressions, the operators paid attention to the subject's mouth and eyes. The subject's eyes were open and the mouth was closed during capture, with the lips gently pressed together to prevent variations in lip posture.

For all situations, the patients were asked to sit in front of a mirror, look forward, and try to position their facial midline with the "true vertical" tape (positioned at the center of the mirror). ${ }^{18}$ An assistant helped position each subject by moving the chair into the camera viewing area (Fig 3). The assistant did not ask the subject to move the head or neck at any time during the initial pictures with or without sensors. Between each acquisition, the volunteer was asked to stand, walk around, and move the chair to minimize bias in the reproducibility of the repeated pictures. For the without-sensor initial and repeated photographs, the patients were simply asked to find their most comfortable positions.

For the third and fourth acquisitions, 3-D Guidance trackSTAR (Ascension Technology, Burlington, Vt), a 3D real-time tracking system consisting of miniaturized 6 degrees of freedom sensors, was used. This is an electromagnetic tracking system where a midrange transmitter generates pulsed direct-current magnetic fields for high-accuracy tracking of the position of the attached minisensors. This system is designed to also be used in surgical navigation systems that follow anatomic bodies, instruments, or devices in the operative scenario. The system provides tracking of actual object positions in relation to the skull base 
and assistance for manipulating the object into the desired configuration. In this study, 3 minisensors of the 3D guidance system were attached to a commercially available band-type hearing protector, ordinarily used for reducing the effects of shop or industrial noise. A hole was made in each auricular part of the protector to fit 2 sensors (each was $1 \mathrm{~mm}$ in outer diameter by $10 \mathrm{~mm}$ in length). The third sensor was attached to the middle of the band (Fig 4). All 3 sensors were plugged into a main unit, which communicated to the computer via a universal serial bus port. Software (Cubes; Ascension Technology Corporation, Burlington, Vt) tracked the real-time coordinates of the 3 attached sensors. The sensors were fitted in the patients' ears, and the band was pushed to the neck. Removing sweatshirts with hoods and tucking in collars and other clothing articles around the neckline facilitated adequate capture of the neck, mandible, and ear. Each of the 3 sensors recorded 6 degrees of freedom in head position: the $\mathrm{x}, \mathrm{y}$, and $\mathrm{z}$ (inferior-superior, posteroanterior, and latero-lateral) distances from the center of the transmitter, and the $\mathrm{x}, \mathrm{y}$, and $\mathrm{z}$ rotational coordinates (roll, pitch, and yaw data) for each patient at the moment of acquisition of the third and fourth pictures. For the photographs captured with sensors, an assistant helped with chair movement and head tilt to reach the same $\mathrm{x}, \mathrm{y}$, and $\mathrm{z}$ positions (in millimeters) and the same pitch, yaw, and roll angulations (in degrees) from the initial (photograph 3 ) to the repeated (photograph 4). It was theorized that the closer the values were for $\mathrm{x}, \mathrm{y}$, and $\mathrm{z}$ between photographs 3 and 4 , the better the reproducibility should be.

After acquisition, each image was loaded into the software 3dMD Patient (3dMD) and exported as a stereolithography (STL) binary file. All STL files were converted to .IV extension by using the STL to SGI Inventor 2.0 (IV) Utility Beta (developed by Reuben Reyes, hitechmex@austin.rr.com). The software cranio-maxilofacial (CMF) application (developed at the M. E. Muller $€$ Institute for Surgical Technology and Biomechanics, University of Bern, Bern, Switzerland, with funding of the computer-aided and imageguided medical interventions (Co-Me) network, http://co-me.ch) was used to locate anatomic landmarks in the 3D photos and to measure landmark distances between acquisitions. ${ }^{19}$ Landmarks were placed by the same operator (L.K.P.) in 8 sites: (1) nasion, (2) tip of nose, (3) subnasale, (4) right lip commissure, (5) left lip commissure, (6) midpoint of upper lip vermilion, (7) midpoint of lower lip vermilion, (8) soft tissue B-point (Fig 5). For 10 subjects, the landmark identification was repeated 3 times to assess intraobserver reliability. Distances between the same landmarks were measured between images 1 and 2 and between images 3 and 4 .

\section{Statistical analysis}

To assess the reliability of landmark identification in the 3D photographs, intraclass correlation coefficients were calculated for each landmark at each coordinate. A mixedeffects analysis of variance (ANOVA) model was used with software (version 9.2; SAS, Cary, NC) to test absolute agreement and consistency.

Descriptive statistics were used to show the mean values and standard deviations of the interlandmark distances on repeated acquisitions of head position with and without the 3D live-tracking sensors. Box plots were used to graphically display the variability of interlandmark distance data. The Student $t$ test was conducted to compare the replicability of head position with and without the sensors. The probability of greater than a 2-mm difference between the 2 methods was calculated.

\section{RESULTS}

To assess the reliability of the identification of the landmarks in the 3D photographs, we used the intraclass correlation coefficient; it showed good to excellent reliability (Table I). 
The average distances between landmarks in acquisitions 1 and 2 (unrestrained head posture without sensors) were $17.43 \mathrm{~mm} \pm 0.32 \mathrm{SD}$ with consistent findings for all landmarks studied. The average distances between landmarks in acquisitions 3 and 4 were $6.17 \mathrm{~mm} \pm$ $0.15 \mathrm{SD}$; these findings were also consistent with all landmarks measured (Table II; Fig 6).

All interlandmark distances were highly significantly different between the 2 methods, with all $\mathrm{P}$ values smaller than 0.01 (Table III). The probabilities that the differences between the 2 methods (without and with sensors) are greater than $2 \mathrm{~mm}$ for each landmark are given in the last column of Table III.

\section{DISCUSSION}

In this study, we evaluated the improvement in reproducibility of unrestrained head positioning with the aid of 3D live-tracking sensors. With the technologies for 3D imaging in the health sciences, it is important to establish accurate and reliable methods for standardizing the acquisition and measurement of the images. Three-dimensional imaging software programs now contain tools for rotation and translation of 3D renderings, volumes, and surfaces, as well as registration of different acquisitions with methods based on landmarks, volumes, or surfaces, but there is no available external reference for head positioning. There is no stable reference structure in $3 \mathrm{D}$ facial photographs for soft-tissue assessments in longitudinal studies. We tested the use of 3D minisensors to approximate the same head position between image acquisitions. The intention was to minimize any error that differences in head position would add to the data; however, at different times, changes in anatomic structures and landmarks can occur, and conventional registration of the images is still required for longitudinal superimpositions.

We attempted to reproduce the unrestrained head position 3 dimensionally using 6 degrees of freedom. When comparing the repeated acquisitions of head position with and without the tracking system in this study, a statistically significant difference was found between the interlandmark distances with the 2 methods. There was also a high probability that the difference between the 2 methods would be greater than $2 \mathrm{~mm}$. The interlandmark distance between repeated acquisitions of head position with the sensors was on average approximately $6 \mathrm{~mm}$ compared with approximately $17 \mathrm{~mm}$ observed without the sensors. These findings show that the sensors aided in the reproducibility of the unrestrained head position.

In this investigation, the interval between photographs was only 10 minutes, and future longitudinal studies could pose greater challenges to the reproducibility of the 3D head position. For this study and for longitudinal assessments, the cameras need to be kept in a separate room with the fixed stands taped to the floor. In a longitudinal study of 2D images acquired at 2-month intervals, Solow and Tallgren ${ }^{10}$ used the subject's "orthoposition," which was obtained in standing subjects with a head holder and images taken at 2-month intervals. They relied on the subject's "self balance": ie, the patient's own feeling of natural head position after extending and flexing the head with decreasing amplitude until a feeling of "natural balance" was achieved. Other longitudinal studies have evaluated reproducibility in head positioning at longer time intervals. Cooke et al ${ }^{12}$ compared different head positioning techniques (with and without ear rods or mirrors) at 3 time intervals. At short intervals between acquisitions (4-10 minutes), the best results were in the group without ear rods with a mirror as the reference; after 3 to 6 months, they found that head position was more reproducible with ear rods. In a later study, Cooke ${ }^{20}$ also found that the reproducibility of natural head position decreases with longer intervals between acquisitions, but the variation on natural head position to the true vertical was still less remarkable than the variation to the true vertical with intracranial references. 
In a previous study attempting to reproduce head orientation in natural head position, Usumez and Orhan ${ }^{13}$ introduced a device with 2 inclinometers attached to a pair of eyeglasses: 1 recorded the pitch, and the other recorded the roll angulation. The drawback of this type of setup is that, if it was to be used in clinical situations, the eyeglasses would interfere with the ability to evaluate the subject without the distraction. In our study, each of the 3 sensors recorded 6 degrees of freedom simultaneously: pitch, roll, and yaw inclinations and distances of the head position to the transmitter. In addition, the headset was worn behind the head and did not distort the subject's facial image.

Our findings suggest that a digital 3D tracking system is a promising tool for the reproducibility of head position, but they also highlighted limitations in current $3 \mathrm{D}$ assessment of treatment outcomes. First, stereophotogrammetry systems and other 3D imaging systems such as CBCT scanners do not allow images to be acquired in natural head position. Superimposition of images acquired at different times relies on the ability to reproduce head position; if traditional means of reproducing head position (such as capturing a person in reproducibility) are not achievable, then determining a new method of acquiring images with a reproducible head position is important. Second, if both 3D photographs and CBCT images are necessary, it would be ideal for both 3D images to be taken simultaneously as has been done in 2D studies with cephalograms and 2D photographs such as that of Solow and Tallgren. ${ }^{10}$ With the current configuration of the 3D stereophotogrammetry imaging equipment, it is not clear how this could be accomplished.

If the use of sensors to achieve reproducible head position is the future, then a more robust headset to hold the sensors is needed. An adjustable and measureable interear distance and a posterior screw that creates a tripod effect with the ear buds would be an improvement to the current headset design. Additionally, the imaging software should be able to acquire the photograph, the CBCT, and the sensor coordinates simultaneously. In this study, 2 software programs were used to measure the coordinates and capture the images. The operator needed to click 1 button for image acquisition and another button for coordinate recording; this created a brief time lag between the 2 clicks. The use of an automated chair that is movable in the anteroposterior and superior-inferior axes would aid in patient positioning.

The ultimate goal would be to have a system that eliminates, or at least significantly decreases, the need for operator-guided patient positioning. Future investigations are needed to improve the use of tracking sensors for standardization of head orientation to allow their use in daily clinical practice. A possible approach to facilitate the use of 3D sensors includes the development of software to capture the patient's head coordinates at the initial acquisition and use this information to relocate the generated surface models of later acquisitions to match the first. Another approach that can already be applied to longitudinal studies is to use the patient's initial head coordinates to assist in reorienting the head to the same position as the initial acquisition, automatically acquiring the picture when this position is achieved. The proposed standardization and recording of head positioning in this study differs from procedures currently allowed by commercial software such as Geomagic (Research Triangle Park, NC), 3dMDVultus (Atlanta, Ga), and InVivo (San Jose, Calif) that use best-fit 3D software to correct some of the above problems, since those software tools do not take head positioning into account.

Additional future development might include having a sound signal to alert the patient when he or she approaches the head orientation of an earlier image that should be matched. Another possibility could be the use of a computer display of the patient's current head position over a semitransparent previous image to help the patient or the operator visualize changes needed to achieve the desired head position. Although it is unrealistic to believe that minisensors can ever achieve perfect reproducibility of head position, there is little doubt 
that this methodology needs to be perfected before it is suitable for important research or clinical use.

\section{CONCLUSIONS}

Reliable head orientation during image capture with 3D photography or CBCT continues to be an important aspect of orthodontic diagnosis, treatment, and subsequent assessment of treatment results. The goal of any system designed to improve an orthodontist's ability to properly orient 3D virtual images is to be able to ascertain and record a repeatable head position. This physiologic goal will greatly improve the value of the 3D digital images that have so dazzled the orthodontic specialty over the last decade. As technology progresses and $3 \mathrm{D}$ imaging supersedes $2 \mathrm{D}$ imaging, and the traditional means of standardizing head position are no longer easily usable, minisensors could become an important aspect of capturing the same head position at different times. Based on the findings of our study, the following conclusions can be made.

1. The use of minisensors improves the repeatability of stereophotogrammetric photographs taken by the $3 \mathrm{dMD}$ camera system.

2. Currently, the use of minisensors does not eliminate the need for registration procedures performed by imaging software for evaluation of like images taken at different times.

Although the use of minisensors is a promising tool for the future, several improvements are required before they can be incorporated practically for research or clinical use.

\section{Acknowledgments}

Supported by Grants DE017727 and DE018962 from University of North Carolina.

\section{REFERENCES}

1. Garson JG. The Frankfort craniometric agreement, with critical remarks thereon. J Anth Inst $\mathrm{Gr} \mathrm{Br}$ Ire. $1885 ; 14: 64-83$.

2. Finlay LM. Craniometry and cephalometry: a history prior to the advent of radiography. Angle Orthod. 1980; 50:312-21. [PubMed: 7006468]

3. Van Loon JAW. A new method for indicating normal and abnormal relations of the teeth to the facial lines. Dent Cosmos. 1915; 57:973-83.

4. Broadbent BH. A new x-ray technique and its application to orthodontia. Angle Orthod. 1931; 1:4566.

5. Hofrath H. Die bedeutung der röntgenfern- und abstandsaufnahme für die diagnostik der kieferanomalien. Fortschr Kieferorthop. 1931; 1:232-9.

6. Downs WB. Analysis of the dentofacial profile. Angle Orthod. 1956; 26:191-212.

7. Moorrees CFA, Kean MR. Natural head position, a basic consideration in the interpretation of cephalometric radiographs. Am J Phys Anthropol. 1958; 16:213-34.

8. Broca M. Sur les projections de la tête, et sur un nouvean procédé de céphalom etrie. Bull Soc Anthropol Paris. 1862; 3:514-44.

9. Vig PS, Showfety KJ, Phillips C. Experimental manipulation of head posture. Am J Orthod. 1980; 77:258-68. [PubMed: 6928735]

10. Solow B, Tallgren A. Natural head position in standing subjects. Acta Odontol Scand. 1971; 29:591-607. [PubMed: 5290983]

11. Luyk NH, Whitfield PH, Wardbooth RP, Williams ED. The reproducibility of the natural head position in lateral cephalometric radiographs. Br J Oral Maxillofac Surg. 1986; 24:357-66.

[PubMed: 2945584] 
12. Cooke MS, Orth D, Wei SHY. The reproducibility of natural head posture-a methodological study. Am J Orthod Dentofacial Orthop. 1988; 93:280-8. [PubMed: 3162636]

13. Usumez $\mathrm{S}$, Orhan M. Inclinometer method for recording and transferring natural head position in cephalometrics. Am J Orthod Dentofacial Orthop. 2001; 120:664-70. [PubMed: 11742312]

14. Raju NS, Prasad KG, Jayade VP. A modified approach for obtaining cephalograms in the natural head position. J Orthod. 2001; 28:25-8. [PubMed: 11254800]

15. Chen CM, Lai S, Tseng YC, Lee KT. Simple technique to achieve a natural head position for cephalography. Br J Oral Maxillofac Surg. 2008; 46:677-8. [PubMed: 18757119]

16. Xia JJ, Gateno J, Teichgraeber JF. New clinical protocol to evaluate craniomaxillofacial deformity and plan surgical correction. J Oral Maxillofac Surg. 2009; 67:2093-106. [PubMed: 19761903]

17. Soncul M, Bamber MA. The reproducibility of the head position for a laser scan using a novel morphometric analysis for orthognathic surgery. Int J Oral Maxillofac Surg. 2000; 29:86-90. [PubMed: 10833142]

18. Lundstrom A, Lundstrom F, Lebret LM, Moorrees CF. Natural head position and natural head orientation: basic considerations in cephalometric analysis and research. Eur J Orthod. 1995; 17:111-20. [PubMed: 7781719]

19. Chapuis J, Schramm A, Pappas I, Hallermann W, Schwenzer-Zimmerer K, Langlotz F, et al. A new system for computer-aided preoperative planning and intraoperative navigation during corrective jaw surgery. IEEE Trans Inf Technol Biomed. 2007; 11:274-87. [PubMed: 17521077]

20. Cooke MS. Five-year reproducibility of natural head posture: a longitudinal study. Am J Orthod Dentofacial Orthop. 1990; 97:489-94. [PubMed: 2353678] 


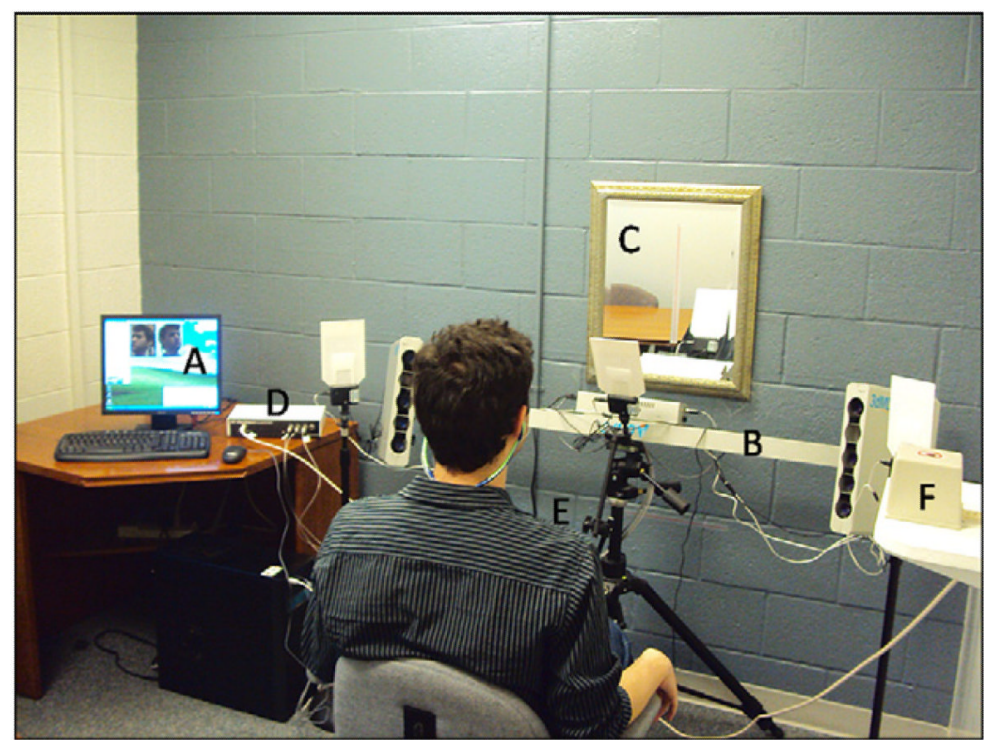

Fig 1.

Computer $(A)$ connected to a multi-lens camera $(B)$ used to capture a 3-D surface image of a patient who was asked to center his face with the red vertical line at the mirror $(C)$. The 3-D real time tracking system developed by Ascension Technology Corporation was composed by a main unit $(D)$, that communicates to the computer providing the coordinates of the sensors $(E)$ which are captured related to a mid-range transmitter $(F)$. 


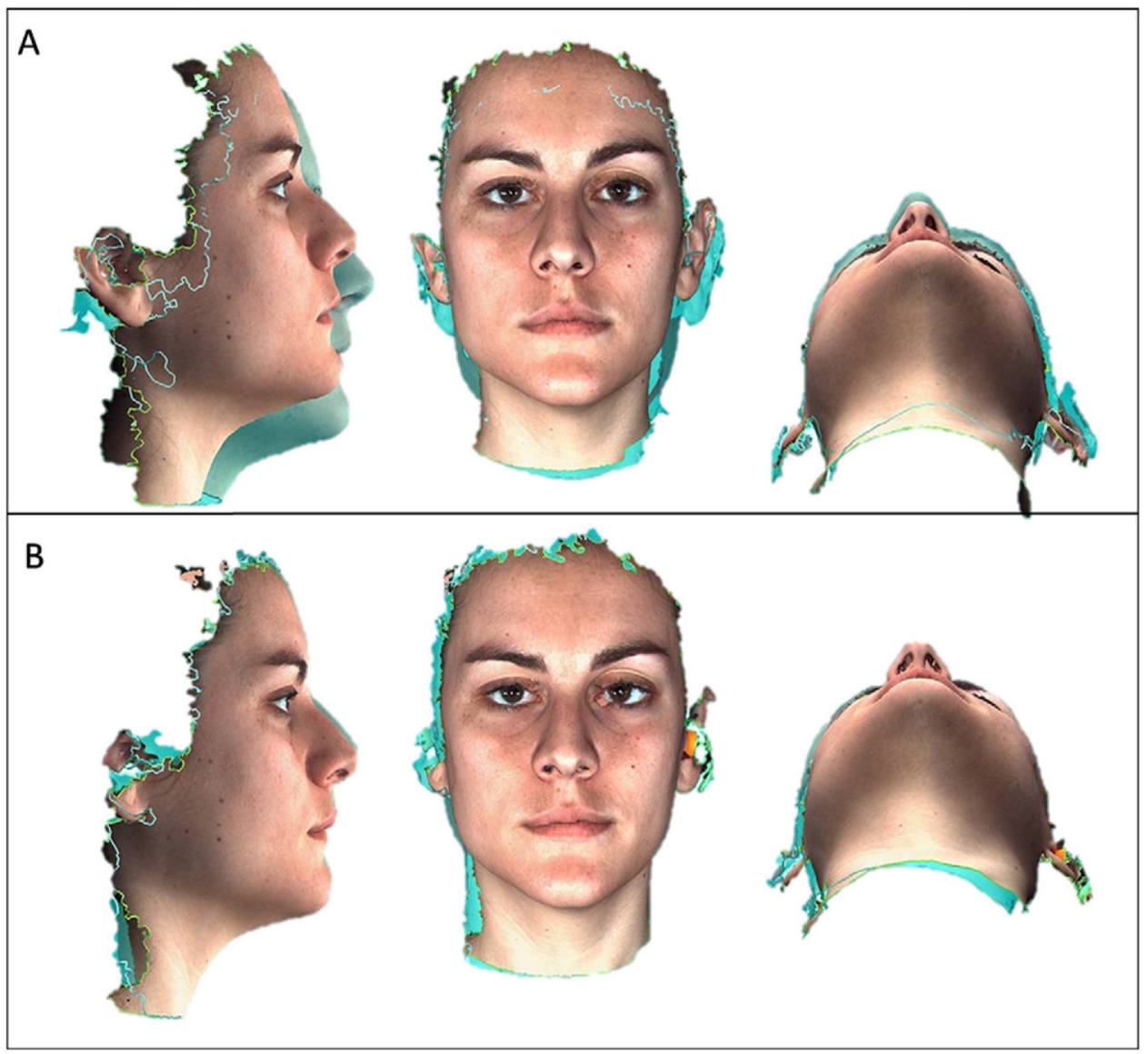

Fig 2.

Three-dimensional surface images overlaid as follows: $\mathbf{A}$, patient in unrestrained head position and the repeated picture (green image); B, patient in unrestrained head position wearing a headset with tracking sensors and the repeated picture (green image). 


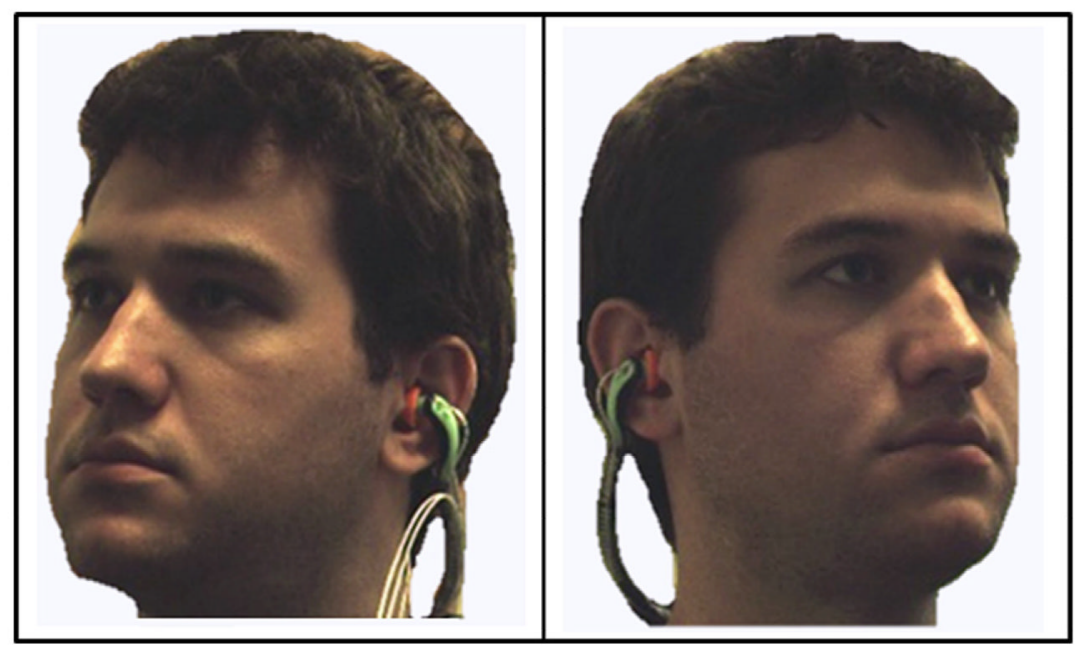

Fig 3.

Computer screen showing a patient with his face well framed in the capture area for the camera acquisitions from the left and right angles. 


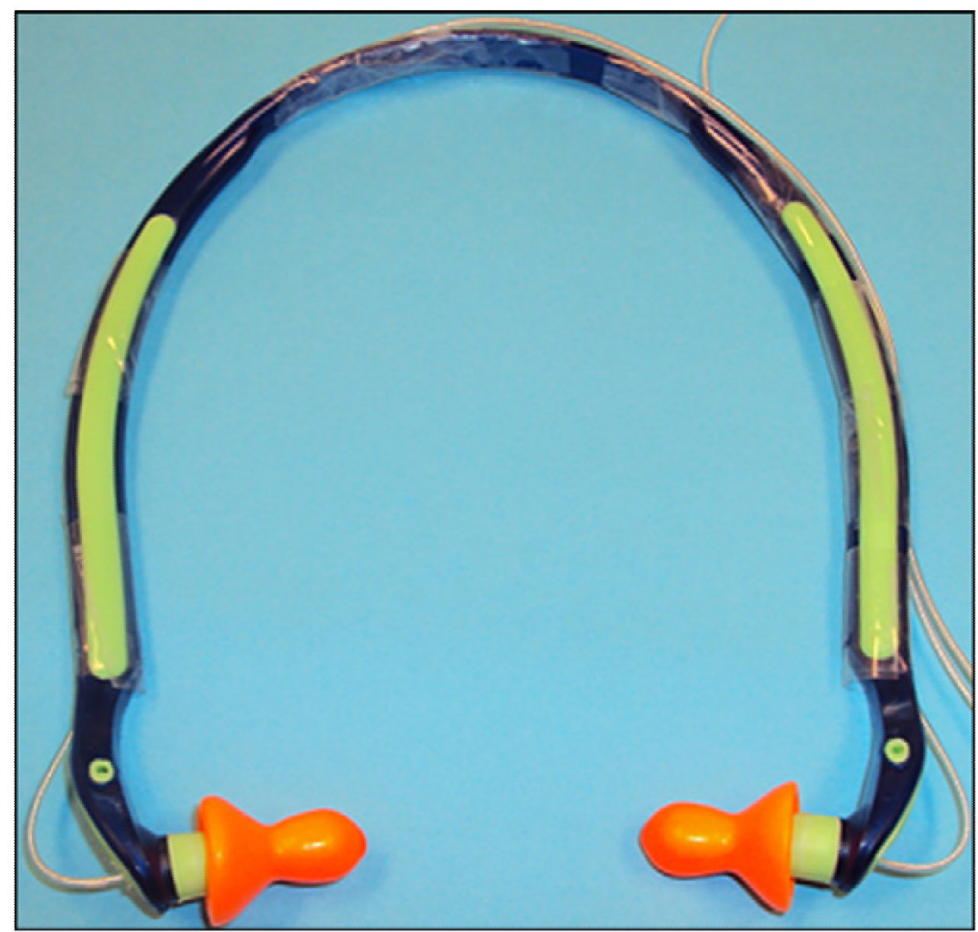

Fig 4.

Sensors were attached to a commercially available band-type hearing protector. A hole was made in each auricular part to fit 2 sensors, and the third was attached to the middle of the band. 


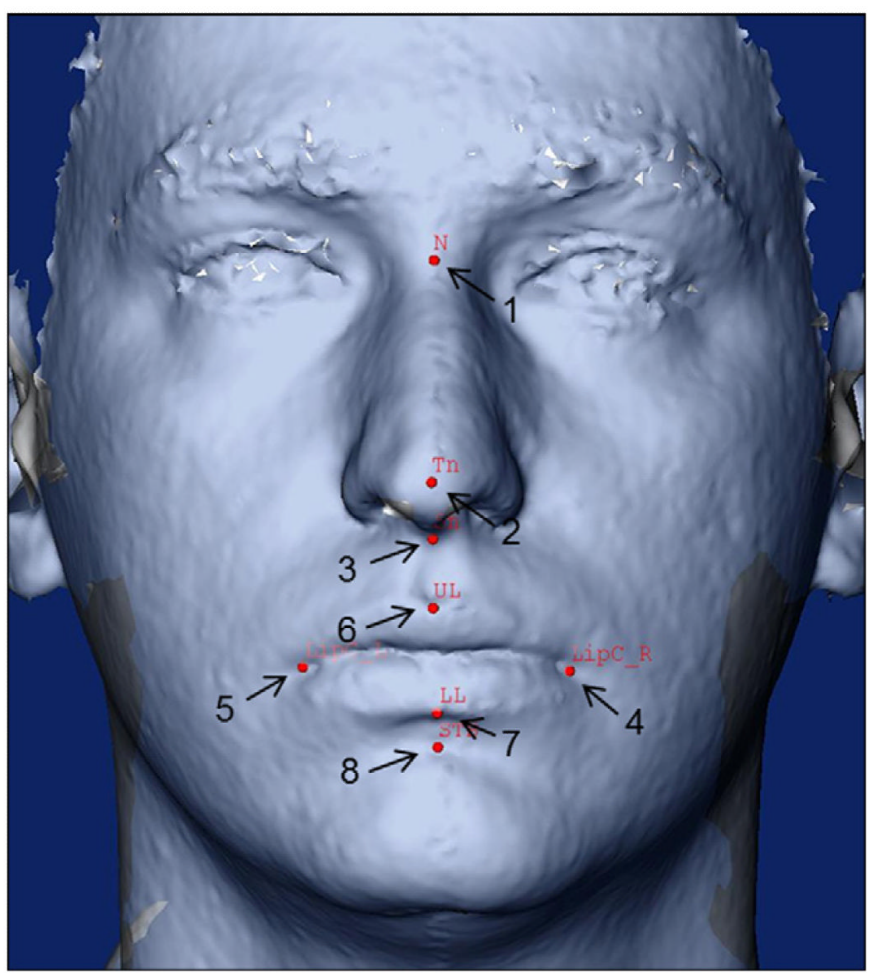

Fig 5.

Landmarks used for evaluation of distances between acquisitions: 1, Nasion; 2, tip of the nose; 3 , subnasale; 4 , right lip commissure; 5 , left lip commissure; 6 , midpoint of the upper lip vermilion; 7 , midpoint of the lower lip vermilion; 8 , soft-tissue B-point. 


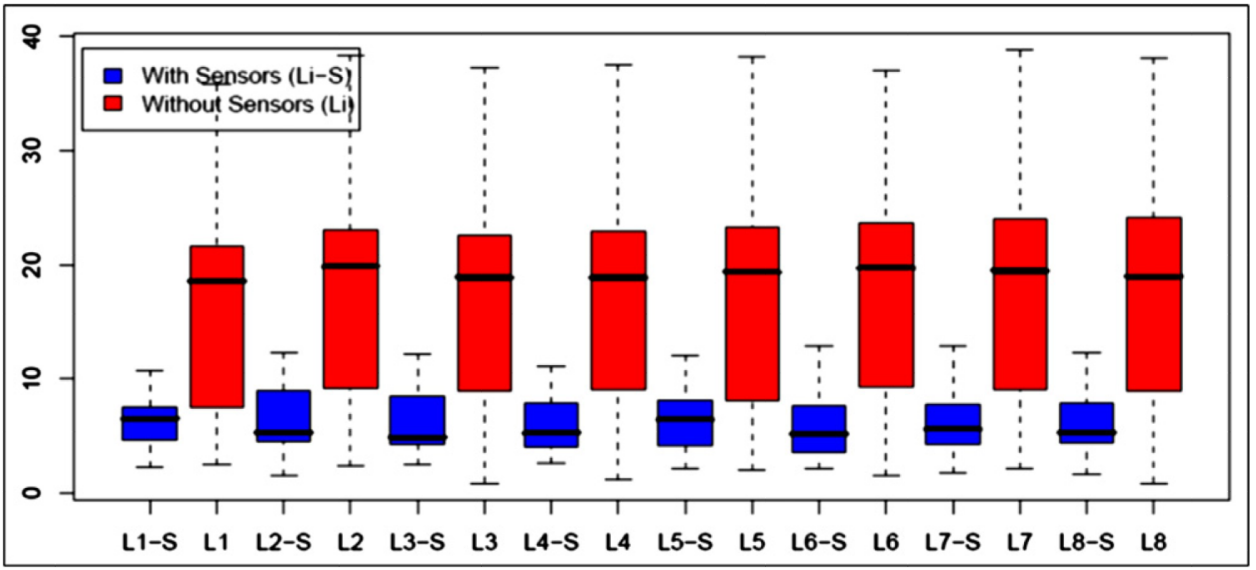

Fig 6.

Box plots showing the variability of interlandmark distance data: $L$, Landmark; $i$, landmark number; $L 1$, left lip commissure; $L 2$, right lip commissure; $L 3$, midpoint of the lower lip vermilion; $L 4$, soft-tissue B-point; $L 5$, nasion; $L 6$, subnasale; $L 7$, tip of the nose; $L 8$, midpoint of the upper lip vermilion. 


\section{Table I}

Reliability estimated by intraclass correlations for each landmark and for each $\mathrm{x}, \mathrm{y}$, and $\mathrm{z}$ coordinate

\begin{tabular}{lccc}
\hline \multirow{2}{*}{ Landmark } & \multicolumn{3}{c}{ Intraobserver reliability } \\
\cline { 2 - 4 } & $\boldsymbol{x}$ & $\boldsymbol{y}$ & $\boldsymbol{z}$ \\
\hline Left lip commissure & 0.97 & 0.99 & 0.99 \\
\hline Right lip commissure & 0.99 & 0.99 & 0.99 \\
\hline Lower lip & 0.60 & 0.54 & 0.99 \\
\hline Nasion & 0.99 & 0.99 & 0.99 \\
\hline Subnasale & 0.85 & 0.99 & 0.99 \\
\hline Soft-tissue B-point & 0.98 & 0.99 & 0.99 \\
\hline Tip of nose & 0.99 & 0.74 & 0.99 \\
\hline Upper lip & 0.99 & 0.99 & 0.99 \\
\hline
\end{tabular}




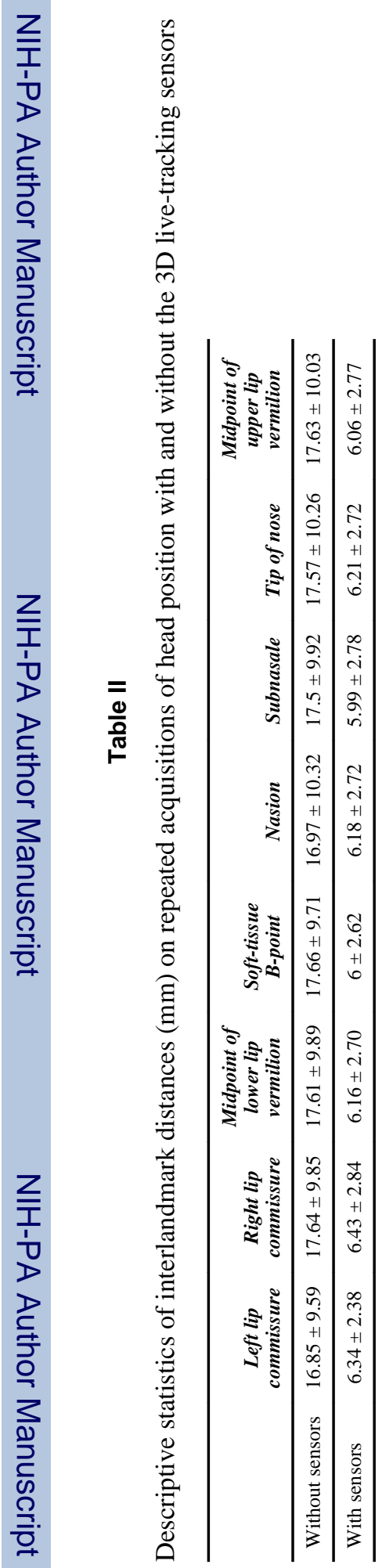




\section{Table III}

Difference between the 2 methods to reproduce head position (without and with sensors): $\mathrm{t}$ statistics, corresponding 2 -sided $P$ values, and the probability that the difference between the 2 methods is greater than 2 $\mathrm{mm}$, assuming that the true mean difference is $0: P(\mathrm{~d}>2 \mathrm{~mm})$

\begin{tabular}{lccc}
\hline $\begin{array}{l}\text { Interlandmark difference } \\
\text { (without sensors-with sensors) }\end{array}$ & $\begin{array}{c}\mathbf{t} \\
\text { statistic }\end{array}$ & $\begin{array}{c}\mathbf{P} \\
\text { value }\end{array}$ & $\begin{array}{c}\mathbf{P} \\
(\boldsymbol{d}>\mathbf{2 ~} \mathbf{~ m m})\end{array}$ \\
\hline Left lip commissure & 4.34 & 0.0004 & 0.21 \\
\hline Right lip commissure & 4.41 & 0.0003 & 0.22 \\
\hline Lower lip & 4.45 & 0.0003 & 0.22 \\
\hline Soft-tissue B-point & 4.62 & 0.0002 & 0.22 \\
\hline Nasion & 4.27 & 0.0004 & 0.22 \\
\hline Subnasale & 4.49 & 0.0002 & 0.22 \\
\hline Tip of nose & 4.35 & 0.0003 & 0.23 \\
\hline Upper lip & 4.51 & 0.0002 & 0.22 \\
\hline
\end{tabular}

